\title{
ON CONTINUOUS LINEAR OPERATORS EXTENDING METRICS
}

\author{
I. STASYUK AND E. D. TYMCHATYN
}

(Communicated by Alexander N. Dranishnikov)

\begin{abstract}
Let $(X, d)$ be a complete metric space. We prove that there is a continuous, linear extension operator from the space of all partial, continuous, bounded metrics with closed, bounded domains in $X$ endowed with the Hausdorff metric topology to the space of all continuous, bounded, metrics on $X$ with the topology of uniform convergence on compact sets. This is a variant of the result of Tymchatyn and Zarichnyi for continuous metrics defined on closed, variable domains in a compact metric space. We get a similar result for the case of continuous real-valued functions.
\end{abstract}

\section{INTRODUCTION}

The theory of extensions of continuous, real-valued functions traces back to the Tietze theorem, while Hausdorff [10] was first to prove a counterpart of Tietze's result for the case of metrics. Hausdorff's theorem on extending a metric defined on a closed subset of a metrizable space to a compatible metric on the whole space was modified and improved by many authors. Dugundji's theorem [9] on continuous, linear extensions of partial, continuous functions defined on a closed subspace of a metric space also has counterparts for metrics. Initially the problem of existence of continuous, linear operators extending (pseudo)metrics was raised and solved for some special cases by Bessaga [8] and was completely solved by Banakh (see [3] and also [4]). Further generalizations of known results on extensions of functions are related to the problem of a simultaneous extension of partial functions with variable domains. In particular, Kunzi and Shapiro 12 proved that there exists a continuous, regular, linear operator extending continuous, real functions defined on compact subsets of a metric space. A variant of the Kunzi-Shapiro result for the noncompact case was obtained in [1] (see also [5]). Tymchatyn and Zarichnyi obtained an analogue of the Kunzi-Shapiro theorem for (pseudo)metrics [15]. They constructed a linear, regular operator extending continuous (pseudo)metrics defined on closed subsets of a compact metrizable space. This operator is continuous with respect to the Hausdorff metric topology on the set of partial (pseudo)metrics where every (pseudo)metric is identified with its graph. Note that the Hausdorff metric convergence of graphs of continuous functions with common domain implies pointwise convergence as well as uniform convergence on compact sets but does not imply the uniform convergence of these functions. However, if the limit function is

Received by the editors October 14, 2010 and, in revised form, November 8, 2011.

2010 Mathematics Subject Classification. Primary 54C20, 54C30; Secondary 54E40.

Key words and phrases. Extension of metrics, continuous linear operator, metric space.

The authors were supported in part by NSERC grant No. OGP 0005616. 
uniformly continuous, then this last implication is true (see [6] and [13]). Of course, all the metrics considered in [15] are uniformly continuous because the initial space is compact, so the convergence of graphs of metrics defined on the whole space is equivalent to the uniform convergence.

In the current paper we obtain a counterpart of the Tymchatyn-Zarichnyi theorem for a noncompact, complete, metric space $X$. We prove that there exists a linear, regular operator extending bounded, continuous pseudometrics defined on closed, bounded subsets of $X$. As in 15] we endow the set of partial pseudometrics with the Hausdorff metric topology, while the set of pseudometrics defined on $X$ is considered with the topology of uniform convergence on compact sets. We show that our operator is continuous and may be improved to preserve metrics. In return, the norm of the operator increases, but it can be chosen so that its norm is arbitrarily close to 1 . We also obtain a different proof of the result from [11. on simultaneous, linear extensions of real functions defined on closed and bounded subsets of a complete metric space.

\section{Preliminaries}

Let $(X, d)$ be a complete metric space with $|X| \geq 2$. Denote by $\exp _{b}(X)$ the set of all closed and bounded subsets of $X$ endowed with the Hausdorff metric $H$ generated by $d$. Recall that the Hausdorff distance between any sets $A, B \in \exp _{b}(X)$ is given by

$$
H(A, B)=\max \left\{\sup _{a \in A} d(a, B), \sup _{b \in B} d(b, A)\right\} .
$$

For every $A \in \exp _{b}(X)$ let $\mathcal{P} \mathcal{M}(A)$ (respectively $\mathcal{M}(A)$ ) stand for the set of all continuous, bounded pseudometrics (respectively metrics) on $A$. We will write $\operatorname{dom} \rho=A$ if $\rho \in \mathcal{P} \mathcal{M}(A)$. It is clear that $\mathcal{P} \mathcal{M}(A)$ and $\mathcal{M}(A)$ are positive cones for every $A \in \exp _{b}(X)$ in the sense that these sets are closed under the operations of pointwise addition and multiplication by a positive number. Let

$$
\mathcal{P} \mathcal{M}=\bigcup\left\{\mathcal{P} \mathcal{M}(A)\left|A \in \exp _{b}(X),\right| A \mid \geq 2\right\}
$$

be the set of all partial, continuous pseudometrics defined on closed and bounded subsets of $X$. Following [15] we endow the set $\mathcal{P} \mathcal{M}$ with the Hausdorff metric topology. We assume that every partial pseudometric $\rho \in \mathcal{P} \mathcal{M}$ is identified with its graph

$$
\Gamma_{\rho}=\{(x, y, \rho(x, y)) \mid x, y \in \operatorname{dom} \rho\},
$$

which is a closed and bounded subset of $X \times X \times \mathbb{R}$. Let $\tilde{d}$ be the box metric on $X \times X \times \mathbb{R}$ and let $\tilde{H}$ denote the Hausdorff metric on $\exp _{b}(X \times X \times \mathbb{R})$ generated by $\tilde{d}$. Then $\mathcal{P} \mathcal{M}$ can be viewed as a subspace of the space $\left(\exp _{b}(X \times X \times \mathbb{R}), \tilde{H}\right)$, where the distance between any two partial metrics from $\mathcal{M}$ is the Hausdorff distance between their graphs. Then the set of partial metrics

$$
\mathcal{M}=\bigcup\left\{\mathcal{M}(A)\left|A \in \exp _{b}(X),\right| A \mid \geq 2\right\}
$$

is a subspace of $\mathcal{P} \mathcal{M}$. For every $\rho \in \mathcal{P} \mathcal{M}$ let $\|\rho\|=\sup \{\rho(x, y) \mid x, y \in \operatorname{dom} \rho\}$.

A map $u: \mathcal{P} \mathcal{M} \rightarrow \mathcal{P} \mathcal{M}(X)$ is called an extension operator if $\left.u(\rho)\right|_{\operatorname{dom} \rho \times \operatorname{dom} \rho}=\rho$ for every $\rho \in \mathcal{P} \mathcal{M}$.

A map $u: \mathcal{P} \mathcal{M} \rightarrow \mathcal{P} \mathcal{M}(X)$ is called linear if $u\left(t_{1} \rho_{1}+t_{2} \rho_{2}\right)=t_{1} u\left(\rho_{1}\right)+t_{2} u\left(\rho_{2}\right)$ for every $t_{1}, t_{2}>0$ and $\rho_{1}, \rho_{2} \in \mathcal{P} \mathcal{M}$ with $\operatorname{dom} \rho_{1}=\operatorname{dom} \rho_{2}$.

A map $u: \mathcal{P} \mathcal{M} \rightarrow \mathcal{P} \mathcal{M}(X)$ is called regular if $\|u(\rho)\|=\|\rho\|$ for every $\rho \in \mathcal{P} \mathcal{M}$. 
Let $I$ denote the unit interval and let $\mathbb{N}$ be the set of positive integers. Also let $\pi_{\{1,2\}}(x, y, A)=(x, y)$ for every $(x, y, A) \in X \times X \times \exp _{b}(X)$. We denote by $\mathbb{R}^{Y}$ the set of all real-valued functions from a topological space $Y$. If $(Y, r)$ is a metric space, then $B_{r}(y, \varepsilon)$ will denote the open ball of radius $\varepsilon$ centered at $y \in Y$.

\section{Auxiliary Results}

For a measurable space $(T, \mathcal{A}, \mu)$ and a Banach space $(E,\|\cdot\|)$, consider the space $L_{1}(T, E)$ of Bochner integrable functions from $T$ to $E$ with the norm defined by $\|\alpha\|=\int_{0}^{1}\|\alpha(t)\| d \mu$ for every $\alpha \in L_{1}(T, E)$.

A set $D$ of measurable functions acting from $(T, \mathcal{A}, \mu)$ into a topological space $Y$ is called decomposable if for every $\alpha, \beta \in D$ and every $C \in \mathcal{A}$ the map $\alpha \chi_{C}+\beta \chi_{T \backslash C}$ belongs to $D$ (here $\chi$ denotes the characteristic function).

A multi-valued map $F: Y \rightarrow Z$ is called lower semicontinuous if the the set $U^{*}=\{y \in Y \mid F(y) \cap U \neq \varnothing\}$ is open in $Y$ for every open subset $U$ of $Z$.

The following selection theorem due to Ageev and Repovs is an important tool in our further construction.

Theorem $3.1([2])$. Let $(T, \mathcal{A}, \mu)$ be a separable, measurable space, E a Banach space, $Y$ a paracompact space and $L_{1}(T, E)$ the space of all Bochner integrable functions from $T$ to $E$. Then every dispersible, multi-valued map $F: Y \rightarrow L_{1}(T, E)$ with closed values admits a continuous selection.

We refer the reader to 2 for the definition of a dispersible multi-valued map and note that we need a slightly weaker variant of the above theorem. We will use the fact that every lower semicontinuous, multi-valued map with decomposable values is dispersible. So if additionally its values are closed, it satisfies the hypothesis of the above theorem (see [2]).

For a topological space $Y$ denote by Meas(Y) the space of all regular, positive, finite Borel measures on $Y$ endowed with the weak topology with respect to $C(Y)$, the Banach space of all continuous and bounded real-valued functions with the sup-norm topology.

For a map $g: Y \rightarrow Z$ a family $\left\{\mu_{z}\right\}_{z \in Z}$ of measures from Meas(Y) is called $a$ fiberwise measure on $g$ if

(i) $\mu_{z}$ continuously depends on $z \in Z$ (this means that $\int_{Y} \varphi d \mu_{z}$ is a continuous function on $Z$ for every $\varphi \in C(Y))$;

(ii) $\operatorname{supp} \mu_{\mathrm{z}}=\bigcap\left\{\mathrm{C} \subset \mathrm{Y} \mid \mathrm{C}\right.$ is closed and $\left.\mu_{\mathrm{z}}(\mathrm{C})=\mu_{\mathrm{z}}(\mathrm{Y})\right\}$ is a subset of $g^{-1}(z)$ for every $z \in Z$.

A map $g$ that admits a probability fiberwise measure is called a Milyutin map. We are going to use the following theorem due to Ageev and Tymchatyn:

Theorem 3.2 ([1]). Let $g: Y \rightarrow Z$ be any map from a complete metric space $Y$ onto a metric space $Z$. Then $g$ is a Milyutin map if and only if there exists a complete subset $Y_{0} \subset Y$ such that $\left.g\right|_{Y_{0}} \rightarrow Z$ is an open surjection.

Finally let us recall some facts on metrization of certain quotient spaces of metric spaces. If $(Y, r)$ is a metric space and $\sim$ is an equivalence relation on $Y$, then one can construct a pseudometric $r^{\prime}$ on $Y / \sim$ as follows. For any equivalence classes $[y]$ and $[z]$ let

$$
r^{\prime}([y],[z])=\inf \left\{r\left(a_{1}, b_{1}\right)+r\left(a_{2}, b_{2}\right)+\cdots+r\left(a_{k}, b_{k}\right)\right\}
$$


where the infimum is taken over all finite collections $a_{1}, a_{2}, \ldots, a_{k}$ and $b_{1}, b_{2}, \ldots, b_{k}$ in $Y$ such that $\left[a_{1}\right]=[y],\left[b_{k}\right]=[z]$ and $\left[b_{i}\right]=\left[a_{i+1}\right]$ for $i \in\{1,2, \ldots, k-1\}$.

Note that if $Y / \sim=Y / B$ where $B$ is a closed subset of $Y$, we obtain a metric on the quotient space defined by $r^{\prime}([y],[z])=\min \{r(y, z), r(y, B)+r(z, B)\}$ for any equivalence classes $[y],[z] \in Y / \sim$.

\section{Extensions of (PSEUdo)METRICS}

The following result is a counterpart of Theorem 4.6 from [15].

Theorem 4.1. Let $(X, d)$ be a complete metric space. There is a linear, regular extension operator $w: \mathcal{P M} \rightarrow \mathcal{P} \mathcal{M}(X)$ which is continuous with respect to the topology of uniform convergence on compact sets on $\mathcal{P} \mathcal{M}(X)$.

Proof. Assume that $(X, d)$ is embedded into a Banach space $(E,\|\cdot\|)$. Recall that $I$ denotes the unit interval with the standard Lebesgue measure and let $L_{1}(I, A)$ be the set of all functions $f \in L_{1}(I, E)$ such that $f(I) \subset A$. Define a multi-valued map $F: X \times \exp _{b}(X) \rightarrow L_{1}(I, E)$ by the formula

$$
F(x, A)=\left\{\begin{array}{l}
\{x\}=L_{1}(I,\{x\}) \text { if } x \in A, \\
L_{1}(I, A) \text { if } x \notin A .
\end{array}\right.
$$

In the same way as in [15, Proposition 4.1], we can prove that $F$ is lower semicontinuous. Also, since $F$ has closed and decomposable values, it is dispersible in the sense of [2] and thus satisfies the hypothesis of Theorem 3.1. Therefore the map $F$ admits a continuous, single-valued selection $f: X \times \exp _{b}(X) \rightarrow L_{1}(I, E)$.

Let $w: \mathcal{P M} \rightarrow \mathbb{R}^{X \times X}$ be given by the formula

$$
w(\rho)(x, y)=\int_{0}^{1} \rho(f(x, \operatorname{dom} \rho)(t), f(y, \operatorname{dom} \rho)(t)) d t
$$

for every $\rho \in \mathcal{P} \mathcal{M}$ and $x, y \in X$. It is easy to check that the map $w$ assigns to every pseudometric $\rho \in \mathcal{P} \mathcal{M}$ a pseudometric $w(\rho)$ on $X$. If $x, y \in \operatorname{dom} \rho$, then by the properties of the selection $f$ we get $f(x, \operatorname{dom} \rho)=\{x\}$ and $f(y, \operatorname{dom} \rho)=\{y\}$. So $w(\rho)(x, y)=\rho(x, y)$, and therefore $w(\rho)$ extends $\rho$ over $X$. Also, it is clear that $w$ is linear and regular. To prove that $w(\rho)$ is a continuous pseudometric on $X$ for every $\rho \in \mathcal{P} \mathcal{M}$ and that the map $w$ is continuous, we will need the following proposition.

Proposition 4.2. For arbitrary sequences $\left\{\rho_{n}\right\}$ in $\mathcal{P} \mathcal{M}$ converging to $\rho \in \mathcal{P} \mathcal{M}$ and $\left\{\left(x_{n}, y_{n}\right)\right\}$ in $X \times X$ converging to $(x, y) \in X \times X$, the sequence $w\left(\rho_{n}\right)\left(x_{n}, y_{n}\right)$ converges to $w(\rho)(x, y)$.

Proof. Let $\left\{\rho_{n}\right\}$ converge to $\rho$ in $\mathcal{P} \mathcal{M}$ and let $\left\{\left(x_{n}, y_{n}\right)\right\}$ converge to $(x, y)$ in $X \times X$. Set $\operatorname{dom} \rho=A, f(x, A)=\alpha, f(y, A)=\beta, \operatorname{dom} \rho_{n}=A_{n}, f\left(x_{n}, A_{n}\right)=\alpha_{n}$ and $f\left(y_{n}, A_{n}\right)=\beta_{n}$ for every $n$.

Let

$$
Q=\left\{\left(a, b, \frac{1}{n}\right) \mid n \in \mathbb{N}, a, b \in A_{n}\right\} \cup\{(a, b, 0) \mid a, b \in A\} .
$$

We assume that $Q$ is endowed with the subspace topology when viewed as a subset of the product $X \times X \times \mathbb{R}$ where $\mathbb{R}$ possesses the standard topology. Define a 
function $h: Q \rightarrow \mathbb{R}$ by the formula

$$
h(a, b, s)=\left\{\begin{array}{l}
\rho(a, b) \text { if } s=0, \\
\rho_{n}(a, b) \text { if } s=1 / n
\end{array}\right.
$$

for every $(a, b, s) \in Q$. Since the sequence $\left\{\Gamma_{\rho_{n}}\right\}$ converges to $\Gamma_{\rho}$ in the Hausdorff metric, the sequence $\left\{A_{n}\right\}$ converges to $A$ in $\exp _{b}(X)$. One can show that if a sequence $\left\{\left(a_{n}, b_{n}\right)\right\}$ in $X \times X$ converges to $(a, b) \in A \times A$ and $\left(a_{n}, b_{n}\right) \in A_{n} \times A_{n}$ for each $n$, then $\rho_{n}\left(a_{n}, b_{n}\right)$ converges to $\rho(a, b)$. Using this condition we conclude that $h$ is a continuous map on $Q$. For every $n$ let $\gamma_{n}: I \rightarrow \mathbb{R}$ be the constant function with value $1 / n$ and let $\gamma: I \rightarrow \mathbb{R}$ be the constant function with value 0 . Then for every $n$ we get

$$
w\left(\rho_{n}\right)\left(x_{n}, y_{n}\right)=\int_{0}^{1} \rho_{n}\left(\alpha_{n}(t), \beta_{n}(t)\right) d t=\int_{0}^{1} h\left(\alpha_{n}(t), \beta_{n}(t), \gamma_{n}(t)\right) d t
$$

and

$$
w(\rho)(x, y)=\int_{0}^{1} \rho(\alpha(t), \beta(t)) d t=\int_{0}^{1} h(\alpha(t), \beta(t), \gamma(t)) d t .
$$

Since the selection $f$ is continuous, we see that $\int_{0}^{1}\left\|\alpha(t)-\alpha_{n}(t)\right\| d t \rightarrow 0$ and $\int_{0}^{1}\left\|\beta(t)-\beta_{n}(t)\right\| d t \rightarrow 0$ as $n \rightarrow \infty$. This implies that $\left\{\alpha_{n}\right\}$ converges to $\alpha$ and $\left\{\beta_{n}\right\}$ converges to $\beta$ in measure on $I$. Also, since $\lim _{n \rightarrow \infty} 1 / n=0$ we see that $\left\{\gamma_{n}\right\}$ converges pointwise to $\gamma$ on $I$. Using the definition of the map $h$ and the fact that the sequence $\left\{\rho_{n}\right\}$ converges to $\rho$ in $\mathcal{P} \mathcal{M}$, we conclude that the sequence of functions $\left\{h\left(\alpha_{n}, \beta_{n}, \gamma_{n}\right)\right\}$ is uniformly bounded on $I$. Suppose that $w\left(\rho_{n}\right)\left(x_{n}, y_{n}\right)$ does not converge to $w(\rho)(x, y)$. Then there is a subsequence $\left(\alpha_{n_{m}}, \beta_{n_{m}}, \gamma_{n_{m}}\right)$ and $c \geq 0$ such that

$$
\int_{0}^{1} h\left(\alpha_{n_{m}}(t), \beta_{n_{m}}(t), \gamma_{n_{m}}(t)\right) d t \underset{n \rightarrow \infty}{\longrightarrow} c \neq \int_{0}^{1} h(\alpha(t), \beta(t), \gamma(t)) d t .
$$

One can find a subsequence $\left(\alpha_{n_{m_{k}}}, \beta_{n_{m_{k}}}, \gamma_{n_{m_{k}}}\right)$ that converges to $(\alpha, \beta, \gamma)$ almost everywhere on $I$. Since $h$ is a continuous map we see that the sequence $h\left(\alpha_{n_{m_{k}}}, \beta_{n_{m_{k}}}, \gamma_{n_{m_{k}}}\right)$ converges to $h(\alpha, \beta, \gamma)$ almost everywhere on $I$. By Lebesgue's dominated convergence theorem we obtain

$$
\int_{0}^{1} h\left(\alpha_{n_{m_{k}}}(t), \beta_{n_{m_{k}}}(t), \gamma_{n_{m_{k}}}(t)\right) d t \underset{n \rightarrow \infty}{\longrightarrow} \int_{0}^{1} h(\alpha(t), \beta(t), \gamma(t)) d t,
$$

a contradiciton. Therefore, $w\left(\rho_{n}\right)\left(x_{n}, y_{n}\right)$ converges to $w(\rho)(x, y)$. This completes the proof of Proposition 4.2

We now continue with the proof of Theorem 4.1. Given any $\rho \in \mathcal{P} \mathcal{M}$ and taking $\rho_{n}=\rho$ for all $n$, we can use Proposition 4.2 to see that $w(\rho)\left(x_{n}, y_{n}\right)$ converges to $w(\rho)(x, y)$ whenever $\left(x_{n}, y_{n}\right)$ converges to $(x, y)$ in $X \times X$. This means that $w(\rho)$ is a continuous pseudometric on $X$ for every $\rho \in \mathcal{P} \mathcal{M}$. Also, the type of convergence discussed in Proposition 4.2, called continuous convergence, in our case is equivalent to the uniform convergence on compact sets on $\mathcal{P} \mathcal{M}(X)$ (see [14, page 109]). So the map $w$ is continuous.

In Theorem 4.1, if $\rho \in \mathcal{M}$ it may happen that $w(\rho)$ is only a pseudometric on $X$ and not a metric. In our next theorem, which is an analogue of Theorem 5.1 from [15], we construct an extension operator for metrics which has all the properties of 
the map $w$ of Theorem 4.1 except that it is not regular. However, we show that this operator can be chosen to have norm arbitrarily close to 1 .

Theorem 4.3. Let $(X, d)$ be a complete metric space. For arbitrary $\eta>0$ there exists a linear extension operator $u: \mathcal{M} \rightarrow \mathcal{M}(X)$ which is continuous with respect to the topology of uniform convergence on compact sets on $\mathcal{M}(X)$ and $\|u(\rho)\| \leq$ $(1+\eta)\|\rho\|$ for every $\rho \in \mathcal{M}$.

Proof. Let $K=\left\{(x, A) \in X \times \exp _{b}(X) \mid x \in A\right\}$. Then $K$ is a closed subset of $X \times \exp _{b}(X)$. Consider the quotient space $\left(X \times \exp _{b}(X)\right) / K$. Let $q: X \times \exp _{b}(X) \rightarrow$ $\left(X \times \exp _{b}(X)\right) / K$ be the corresponding quotient map. Using the method of constructing a metric on a quotient space discussed in Section 3. we define a metric $r$ on $\left(X \times \exp _{b}(X)\right) / K$ by the formula

$$
r([(x, A)],[(y, B)])=\min \{\min \{d(x, y)+H(A, B), d(x, A)+d(y, B)\}, 1\}
$$

for every $(x, A),(y, B) \in X \times \exp _{b}(X)$.

Now let $L=\left\{(x, y, A) \in X \times X \times \exp _{b}(X) \mid x, y \in A, x \neq y\right\}$ and $L^{\prime}=$ $\left\{(x, y, A) \in X \times X \times \exp _{b}(X) \mid x, y \in A\right\}$.

Since $(X, d)$ is a complete space, so is $\left(\exp _{b}(X), H\right)$ (see [7, Theorem 3.2.4]) and, therefore, so is $X \times X \times \exp _{b}(X)$ with the box metric. It is easy to see that $L^{\prime}$ is a closed subspace of $X \times X \times \exp _{b}(X)$. So $L^{\prime}$ is complete. To show that $L$ is open in $L^{\prime}$, let $(x, y, A) \in L$ be arbitrary and let $d(x, y)=s>0$. Then for instance the set

$$
\left(B_{d}(x, s / 3) \times B_{d}(y, s / 3) \times B_{H}(A, s / 3)\right) \cap L^{\prime}
$$

is an open neighborhood of $(x, y, A)$ in $L$. We conclude that the space $L$ is completely metrizable since it is an open subspace of a complete space.

Let $\pi: L \rightarrow \exp _{b}(X)$ be defined by $\pi(x, y, A)=A$ for $(x, y, A) \in L$. Then by Theorem $3.2 \pi$ is a Milyutin map because it is an open surjection and $L$ is completely metrizable. Therefore, one can find a continuous family $\left\{\mu_{B}\right\}_{B \in \exp _{b}(X)}$ of probability measures on $L$ with support of $\mu_{B}$ contained in $\pi^{-1}(B)$ for each $B \in \exp _{b}(X)$. Define a map $v: \mathcal{M} \rightarrow \mathbb{R}$ by the formula

$$
v(\rho)=\int_{\pi^{-1}(\operatorname{dom} \rho)} \rho\left(\pi_{\{1,2\}}(a, b, \operatorname{dom} \rho)\right) d \mu_{\operatorname{dom} \rho}
$$

for every $\rho \in \mathcal{M}$. Note that $v(\rho)>0$ for every partial metric $\rho \in \mathcal{M}$. Also, $v$ is linear, that is, $v\left(t_{1} \rho_{1}+t_{2} \rho_{2}\right)=t_{1} v\left(\rho_{1}\right)+t_{2} v\left(\rho_{2}\right)$ for every $t_{1}, t_{2}>0$ and $\rho_{1}, \rho_{2} \in \mathcal{M}$ with $\operatorname{dom} \rho_{1}=\operatorname{dom} \rho_{2}$.

Now let $u: \mathcal{M} \rightarrow \mathbb{R}^{X \times X}$ be defined as follows:

$$
u(\rho)(x, y)=w(\rho)(x, y)+v(\rho) \cdot r(q(x, \operatorname{dom} \rho), q(y, \operatorname{dom} \rho))
$$

for every $\rho \in \mathcal{M}$ and $x, y \in X$, where $w$ is the map constructed in Theorem 4.1 and $r$ is the metric on the quotient space $\left(X \times \exp _{b}(X)\right) / K$ defined above.

Let $\rho \in \mathcal{M}$ be arbitrary. To see that $u(\rho)$ is a metric on $X$ extending $\rho$, note first that $u(\rho)$ is symmetric and satisfies the triangle inequality condition because $u(\rho)$ is a sum of two pseudometrics on $X$. If $x, y \in \operatorname{dom} \rho$ we obtain $q(x, \operatorname{dom} \rho)=$ $q(y, \operatorname{dom} \rho)$, and therefore $u(\rho)(x, y)=w(\rho)(x, y)+v(\rho) \cdot 0=\rho(x, y)$. So $u(\rho)$ is an extension of $\rho$ over $X$. Now for any distinct points $x, y \in X$ that do not both belong to $\operatorname{dom} \rho$, we get $u(\rho)(x, y)>0$ because $v(\rho)>0$ and $q(x, \operatorname{dom} \rho) \neq q(y, \operatorname{dom} \rho)$, which implies $r(q(x, \operatorname{dom} \rho), q(y, \operatorname{dom} \rho))>0$. So $u(\rho)$ is a metric on $X$ extending $\rho$ for every $\rho \in \mathcal{M}$. Using the definition of the map $u$ and the fact that $w$ and $v$ are 
linear, we see that $u$ is linear as well. To prove that $u(\rho)$ is continuous for every $\rho \in \mathcal{M}$ and that $u$ is a continuous map, we need the following proposition.

Proposition 4.4. For arbitrary sequences $\left\{\rho_{n}\right\}$ in $\mathcal{M}$ converging to $\rho \in \mathcal{M}$ and $\left\{\left(x_{n}, y_{n}\right)\right\}$ in $X \times X$ converging to $(x, y) \in X \times X$, the sequence $\left\{u\left(\rho_{n}\right)\left(x_{n}, y_{n}\right)\right\}$ converges to $u(\rho)(x, y)$.

Proof. Let $\left\{\rho_{n}\right\}$ converge to $\rho$ in $\mathcal{M}$ and let $\left\{\left(x_{n}, y_{n}\right)\right\}$ converge to $(x, y)$ in $X \times X$. Set $\operatorname{dom} \rho=A$, and $\operatorname{dom} \rho_{n}=A_{n}$ for every $n$. Then

$$
u(\rho)(x, y)=w(\rho)(x, y)+v(\rho) \cdot r(q(x, A), q(y, A))
$$

and

$$
u\left(\rho_{n}\right)\left(x_{n}, y_{n}\right)=w\left(\rho_{n}\right)\left(x_{n}, y_{n}\right)+v\left(\rho_{n}\right) \cdot r\left(q\left(x_{n}, A_{n}\right), q\left(y_{n}, A_{n}\right)\right)
$$

for every $n \in \mathbb{N}$. We have already proved in Proposition 4.2 that $w\left(\rho_{n}\right)\left(x_{n}, y_{n}\right)$ converges to $w(\rho)(x, y)$. Since $r$ and $q$ are continuous we see that $r\left(q\left(x_{n}, A_{n}\right), q\left(y_{n}, A_{n}\right)\right)$ converges to $r(q(x, A), q(y, A))$ as $n \rightarrow \infty$. To check whether $v\left(\rho_{n}\right)$ converges to $v(\rho)$, consider two cases.

Case 1. Let $A_{n}=A$ for every $n$. Since the metrics $\rho$ and $\left\{\rho_{n}\right\}$ have common domain and since $\left\{\Gamma_{\rho_{n}}\right\}$ converges to $\Gamma_{\rho}$ in the Hausdorff distance, we conclude that $\left\{\rho_{n}\right\}$ converges to $\rho$ pointwise on $A \times A$ and therefore the sequence $\left\{\rho_{n} \circ \pi_{\{1,2\}}\right\}$ is uniformly bounded and converges pointwise to $\rho \circ \pi_{\{1,2\}}$ on $L$. By Lebesgue's dominated convergence theorem we obtain

$$
v\left(\rho_{n}\right)=\int_{\pi^{-1}(A)} \rho_{n}\left(\pi_{\{1,2\}}(a, b, A)\right) d \mu_{A} \underset{n \rightarrow \infty}{\longrightarrow} \int_{\pi^{-1}(A)} \rho\left(\pi_{\{1,2\}}(a, b, A)\right) d \mu_{A}=v(\rho) .
$$

Case 2. Let $A_{n} \neq A$ for every $n \in \mathbb{N}$. Then passing to a subsequence if necessary we may also assume that $A_{n} \neq A_{k}$ for $n \neq k$. Let

$$
Z=\left\{(a, b, C) \mid C \in\left\{A, A_{1}, A_{2}, \ldots\right\}, a, b \in C, a \neq b\right\}
$$

be considered as a subspace of $L$. Define a map $p: Z \rightarrow \mathbb{R}$ by the formula

$$
p(a, b, C)=\left\{\begin{array}{l}
\rho(a, b) \text { if } C=A \\
\rho_{n}(a, b) \text { if } C=A_{n} .
\end{array}\right.
$$

Then as in Proposition 4.2 one can show that the map $p$ is continuous on $Z$, and since the family of measures $\left\{\mu_{A_{n}}\right\}_{n=1}^{\infty} \cup\left\{\mu_{A}\right\}$ is continuous, we obtain

$$
\begin{gathered}
v\left(\rho_{n}\right)=\int_{\pi^{-1}\left(A_{n}\right)} \rho_{n}\left(\pi_{\{1,2\}}\left(a, b, A_{n}\right)\right) d \mu_{A_{n}}=\int_{Z} p(a, b, C) d \mu_{A_{n}} \\
\underset{n \rightarrow \infty}{\longrightarrow} \int_{Z} p(a, b, C) d \mu_{A}=\int_{\pi^{-1}(A)} \rho\left(\pi_{\{1,2\}}(a, b, A)\right) d \mu_{A}=v(\rho) .
\end{gathered}
$$

Therefore, $u\left(\rho_{n}\right)\left(x_{n}, y_{n}\right) \rightarrow u(\rho)(x, y)$ as $n \rightarrow \infty$.

To prove the convergence of $u\left(\rho_{n}\right)\left(x_{n}, y_{n}\right)$ to $u(\rho)(x, y)$ for any sequences $\left\{\rho_{n}\right\}$ and $\left\{\left(x_{n}, y_{n}\right)\right\}$, we will have to pass to subsequences and apply Cases 1 and 2 .

We continue with the proof of Theorem 4.3. Using Proposition 4.4 and similar reasoning to that in Theorem 4.1, we conclude that $u(\rho)$ is a continuous metric on $X$ for every $\rho \in \mathcal{M}$ and that $u$ is a continuous operator with respect to the topology of uniform convergence on compact sets on $\mathcal{M}(X)$. 
To verify the last condition of the theorem note that $\|\rho\| \leq\|u(\rho)\|$ for every $\rho \in \mathcal{M}$, but since $r$ is bounded by 1 , for every $\eta>0$ preserving all the above properties we can construct a modification $u_{\eta}$ of the extension operator $u$ defined as follows:

$$
u_{\eta}(\rho)(x, y)=w(\rho)(x, y)+\eta \cdot v(\rho) \cdot r(q(x, \operatorname{dom} \rho), q(y, \operatorname{dom} \rho)) .
$$

Then $\left\|u_{\eta}(\rho)\right\| \leq\|\rho\|+\eta\|\rho\|=(1+\eta)\|\rho\|$.

\section{EXTENSIONS OF FUnCTIONS}

For every $A \in \exp _{b}(X)$ let $C^{*}(A)$ denote the family of continuous, bounded, real-valued functions on $A$ and let $C_{b}^{*}=\bigcup\left\{C^{*}(A) \mid A \in \exp _{b}(X)\right\}$. As before we assume that each $g \in C_{b}^{*}$ is identified with its graph and the distance between partial functions is the Hausdorff distance between their graphs in $\exp _{b}(X \times \mathbb{R})$. The set of continuous, bounded functions on $X$ is denoted by $C^{*}(X)$. Using the same reasoning as in Theorem 4.1 we obtain a different proof of the main result from [1]:

Theorem 5.1. Let $(X, d)$ be a complete metric space. There exists a regular, linear extension operator $e: C_{b}^{*} \rightarrow C^{*}(X)$. This operator is continuous with respect to the topology of uniform convergence on compact sets on $C^{*}(X)$.

Proof. Let $f$ be a continuous selection constructed in the proof of Theorem 4.1. Then for every $g \in C_{b}^{*}$ and $x \in X$ we set $e(g)(x)=\int_{0}^{1} g(f(x, \operatorname{dom} g)(t)) d t$. The properties of the map $e$ can be verified in the same way as in Theorem 4.1 .

\section{ACKNOWLEDGEMEnT}

The authors wish to express gratitude to the referee for useful remarks which helped to improve the paper.

\section{REFERENCES}

[1] S. Ageev, E.D. Tymchatyn, On exact Milyutin maps, Topology Appl. 153 (2005), 227-238. MR2175347 (2006h:54008)

[2] S. Ageev, D. Repovs, On selection theorems with decomposable values, Topol. Methods Nonlinear. Anal. 15 (2000), 385-399. MR.1784148 (2002a:54012)

[3] T. Banakh, AE(0)-spaces and regular operators extending (averaging) pseudometrics, Bull. Pol. Acad. Sci. Math. 42 (1994), no. 3, 197-206. MR1811849

[4] T. Banakh, C. Bessaga, On linear operators extending [pseudo]metrics, Bull. Pol. Acad. Sci. Math. 48 (2000), no. 1, 35-49. MR.1751152 (2001e:54023)

[5] T. Banakh, N. Brodskiy, I. Stasyuk, E.D. Tymchatyn, On continuous extension of uniformly continuous functions and metrics, Colloq. Math. 116 (2009), 191-202. MR2520139 (2011b:54037)

[6] G. Beer, More on convergence of continuous functions and topological convergence of sets, Canad. Math. Bull. 28 (1985) no. 1, 52-59. MR778261(86e:54018)

[7] G. Beer, Topologies on Closed and Closed Convex Sets, Kluwer Academic, Dordrecht, 1993. MR 1269778 (95k:49001)

[8] C. Bessaga, On linear operators and functors extending pseudometrics, Fund. Math. 142 (1993), no. 2, 101-122. MR.1211761 (94h:54033)

[9] J. Dugundji, An extension of Tietze's theorem, Pacific J. Math. 1 (1951), 353-367. MR.0044116 (13:373c)

[10] F. Hausdorff, Erweiterung einer Homöomorphie, Fund. Math. 16 (1930), 353-360.

[11] A. Koyama, I. Stasyuk, E.D. Tymchatyn, A. Zagorodnyuk, Continuous linear extensions of functions, Proc. Amer. Math. Soc. 138 (2010), 4149-4155. MR2679637 (2011h:54022) 
[12] H.P. Kunzi, L.B. Shapiro, On simultaneous extension of continuous partial functions, Proc. Amer. Math. Soc. 125 (1997), 1853-1859. MR1415348(98g:54015)

[13] S.A. Naimpally, Graph topology for function spaces, Trans. Amer. Math. Soc. 123 (1966), 267-272. MR0192466 (33:691)

[14] L. Narici, E. Beckenstein, Topological Vector Spaces, Pure and Applied Mathematics 95, Marcel Dekker Inc., New York-Basel, 1985. MR812056 (87c:46003)

[15] E. D. Tymchatyn, M. Zarichnyi, On simultaneous linear extensions of partial (pseudo) metrics, Proc. Amer. Math. Soc. 132 (2004), 2799-2807. MR2054807 (2005d:54032)

Department of Mechanics and Mathematics, Lviv National University, UniversytetSKA Str. 1, Lviv, 79000, Ukraine

Current address: Department of Computer Science and Mathematics, Nipissing University, 100 College Drive, Box 5002, North Bay, ON, 51B 8L7, Canada

E-mail address: i_stasyuk@yahoo.com

Department of Mathematics and Statistics, McLean Hall, University of SaskatcheWAN, 106 Wiggins RoAd, SASkatoon, SK S7N 5E6, CANAdA

E-mail address: tymchat@math.usask.ca 